\title{
Myelin water fraction decrease in mild traumatic brain injury
}

\author{
Bretta Russell-Schulz ${ }^{1}$, Irene Vavasour ${ }^{1}$, Jing Zhang ${ }^{2}$, Alex L. MacKay ${ }^{1,3}$, Victoria Purcell ${ }^{4}$, \\ Angela M. Muller ${ }^{5}$, Leyla Brucar ${ }^{5}$, Ivan J. Torres ${ }^{2,4}$, William Panenka ${ }^{2,4}$, Naznin Virji-Babul ${ }^{5,6^{*+}}$ \\ ${ }^{1}$ Department of Radiology, University of British Columbia, Vancouver, BC, Canada \\ ${ }^{2}$ BC Children's Hospital Research Institute, Vancouver, BC, Canada \\ ${ }^{3}$ Department of Physics and Astronomy, University of British Columbia, Vancouver, BC, Canada \\ ${ }^{4}$ Department of Psychiatry, University of British Columbia, Vancouver, BC, Canada \\ ${ }^{5}$ Djavad Mowafaghian Centre for Brain Health, University of British Columbia, Vancouver, BC, \\ Canada \\ ${ }^{6}$ Department of Physical Therapy, University of British Columbia, Vancouver, BC, Canada \\ *Correspondence: \\ Naznin Virji-Babul, PT, Ph.D \\ Email: naznin.virji-babul@ubc.ca \\ ${ }^{+}$Department of Physical Therapy \\ University of British Columbia \\ 212 - 2177 Wesbrook Mall \\ Vancouver, B.C., V6T 1 Z3 \\ Canada \\ Tel: 1-604-827-4966 \\ Fax: 1-604-822-1870
}

Word Count: 3656

Figure Number: 5

Keywords : Magnetic Resonance Imaging, Mild Traumatic Brain Injury, Myelin, Cognition, White Matter, Myelin Water Fraction, Chronic Injury

\begin{abstract}
The increased incidence of reported traumatic brain injury (TBI) and its potentially serious long-term consequences have enormous clinical and societal impacts. The diffuse and continually evolving secondary changes after TBI make it challenging to evaluate the changes in brain-behaviour relationships. In this study we used myelin water imaging to evaluate changes in myelin water fraction (MWF) in individuals with chronic brain injury and evaluated their cognitive status using the NIH Toolbox Cognitive Battery. Twenty-two adults with mild or severe brain injury and twelve age, gender and education matched healthy controls took part in this study. We found a significant decrease in global white matter MWF in individuals with mild TBI compared to the healthy controls. Significantly lower MWF was evident in most white matter ROIs examined including the corpus callosum (separated into genu, body and splenium), minor forceps, right anterior thalamic radiation, left inferior longitudinal fasciculus; and right and left superior longitudinal fasciculus and corticospinal tract. No significant correlations were found between MWF in mild TBI and the cognitive measures. These results show for the first time the loss of myelin in chronic mild TBI.
\end{abstract}




\section{Introduction}

47 Every year about 160,000 Canadians sustain brain injuries, the most prevalent causes being falls and 48 motor vehicle accidents (MVA) (Brain Injury Canada). An impact to the head results in an immediate and direct insult to the brain, setting off a complex cascade of metabolic and neurochemical events. These effects can lead to long-term changes in brain physiology leading to cognitive, motor and affective dysfunction (1-3). Over a lifetime, repeated brain trauma or a single moderate or severe TBI is associated with an increased incidence of multiple neuropsychiatric conditions and is a significant risk factor for developing neurodegenerative disorders $(4,5)$. The diffuse and continually evolving secondary changes that are the hallmark of TBI have made it extremely challenging to evaluate brainbehavior relationships. Neurodegeneration has been found in the chronic phases after TBI(6) and detected using atrophy measures, however conventional neuroimaging tools (such as CT and MRI) cannot detect the widespread and often subtle changes in structure and function that occur in the brain following TBI (7). The long-term effects of a mild TBI (mTBI) are less clear than more severe head injuries and neuroimaging findings are less consistent $(8,9)$. Thus advanced MR imaging techniques are increasingly being used to examine and monitor changes in the brain following TBI (10-12).

60

61

62

63

64

65

66

67

Physiologically, movement of the brain within the skull causes shearing/stretching of the axons and initiates a cascade of molecular events which disrupts normal brain cell function. Metabolic changes occur rapidly following axonal strain, altering the permeability of sodium channels, resulting in an increase in intra-axonal calcium and ultimately a failure of the sodium pump, causing further metabolic disruptions (13). Long white matter tracts are particularly vulnerable to these forces and diffuse axonal injury (DAI) can lead to demyelination and axonal loss resulting in brain atrophy $(5,14-18)$. A long term follow-up study of individuals with severe TBI found neurodegeneration in the chronic phase as measured by volume reduction in the corpus callosum between 1-8 years post injury (19). Loss of myelin increases axonal vulnerability to further trauma and may predispose the axon to further damage $(20,21)$. In animal models of TBI, extensive demyelination is consistently reported $(22,23)$, and human post mortem studies have found evidence of loss of myelin (24) years after the initial TBI symptoms have resolved. Given the importance of monitoring white matter tracts in TBI, a validated quantitative technique to examine myelination (loss and possible remyelination $(20,25)$ ) of these tracts would provide a powerful approach to understand the pathology of TBI, the evolving nature of the injury and ultimately could be used to evaluate the impact of neurorehabilitation.

Measuring myelin in vivo using MR is nontrivial and requires specialized techniques (26,27). Myelin is a lipid rich membrane assembly that wraps around axons in a series of layers. Myelin enables saltatory conduction which produces fast and efficient neuronal signal transmission across the brain and throughout the body. The loss or disruption of myelin results in loss of saltatory conduction causing slower signal propagation and thus delay of translation of information. Measuring non-aqueous protons in myelin is difficult because the MR signal decays too rapidly to be measured by common MR techniques. The water trapped between myelin bilayers is known as myelin water and it is accessible using MR. Several different techniques have been developed to measure the myelin water (28), one such approach involves using multi-component T2 analysis, myelin water imaging (MWI). Multicomponent $\mathrm{T} 2$ relaxation can be used to detect different water environments in human brain tissue (2931 ), including a short $\mathrm{T} 2$ component that is thought to be myelin water (32-40). The proportion of water in brain that can be attributed to myelin water is known as the myelin water fraction (MWF) which can be used as a marker for myelin (41). MWF has been validated as a myelin marker in histological studies in animal models (42-44) and human post-mortem multiple sclerosis brain (45-47) 
and spinal cord (48). MWF has been found to be decreased in normal appearing white matter in neurodegenerative diseases such as multiple sclerosis (49-52). MWF has previously been shown to decrease post single sports concussion (mTBI) and then recover after 2 months (53), showing the utility of MWF in mTBI.

It should be noted that there are other factors aside from pathology that can cause changes to myelination and thus MWF. In humans, myelin development begins in utero and develops quickly through the first few years but continues to increase through adolescence and young adulthood (54) and is thought to increase with learning in healthy adult brains (55). MWF in healthy individuals is significantly correlated with increasing age and years of education $(56,57)$. A subsequent study (58) confirmed these significant correlations with healthy control MWF but also found age and education relationships in a cohort of first-episode schizophrenia patients.

Cognitive decline is a common consequence of moderate/severe TBI and can continue long-term (59). In most cases of mTBI, there is little to no evidence of long-term cognitive impairment(60-63), however a portion of patients with mTBI will report persistent symptoms, such as post-concussive syndrome and other cognitive function issues(64-69). It is important to characterize the cognitive function of TBI subjects as it is a potent predictor of functional capacity, and targeted rehabilitation measures are available. A number of groups have documented an association between myelin water fraction and cognition. Lang et al.(58) showed that Frontal white matter MWF correlated with scores on a premorbid IQ test, the North American Adult Reading Test (NAART) and Choi et. al. (70) found a correlation between a processing speed index and a global measure of apparent myelin water fraction in a group of moderate to severe TBI subjects 3 months post-injury. These findings show that myelin measures may reflect cognitive functional changes in TBI.

Here we examine myelin water fraction (MWF) within a heterogeneous group of chronic TBI participants and compare mild TBI MWF to healthy controls using a whole cerebrum coverage MWF sequence to characterize differences in myelin. We also examined the effects of age and years of education on MWF, independent of TBI. Finally, we compared cognitive scores between the two groups and their relationship with MWF. Based on results from a previous study in TBI (70) we hypothesized that MWF would be particularly associated with fluid cognitive abilities.

\section{Methods}

\subsection{Subject demographics}

Participants were recruited from brain injury associations across the Greater Vancouver area as part of a study to evaluate the impact of an intensive cognitive intervention program (Arrowsmith) in adults with TBI (71). All healthy controls were recruited from the lower mainland of Vancouver in close proximity to the university. All controls were screened to ensure that they had no history of head trauma, neuropsychiatric disorders, or any other neurological conditions. The participants with TBI were first interviewed to determine eligibility and to evaluate the severity of TBI. Participants were excluded if they were currently in litigation or if they had other severe medical conditions affecting brain function. They were also excluded if they had a diagnosis of psychiatric illness based on a Miniinternational neuropsychiatric interview (MINI) (72). The severity of injury was based on self-report using the diagnostic criteria from the World Health Organization (WHO). Information was retrospectively reported by the subjects, where mild TBI was defined as Loss of Consciousness (LOC) 
137 of less than 30 minutes and Post-traumatic amnesia (PTA) less than 24 hours, and severe TBI was defined as LOC over 24 hours. All participants provided written consent according to the guidelines set forth by the Clinical Research Ethics Board at the University of British Columbia. Subject demographics are given in Table 1, and individual subject TBI history and severity are given in Supplementary Table S1. Time since injury varied from 6 months to 28 years post injury across subjects, and 6 months was assumed to be in the chronic stage for mTBI(73).

\subsection{MRI protocol}

145

146

147

MRI scans were completed on a 3T Philips Achieva scanner. Sequences included a 3DT1 structural scan (MPRAGE TR=3000 ms, TI $=1072 \mathrm{~ms}, 1 \times 1 \times 1 \mathrm{~mm}^{3}$ voxel, 160 slices) for registration and segmentation of global white matter and white matter regions of interest (ROIs) and a 3D 48-echo Gradient and Spin Echo (GRASE) T2 relaxation sequence with an EPI factor of 3 (TR=1073, echo spacing $=8 \mathrm{~ms}, 20$ slices acquired at $1 \times 2 \times 5 \mathrm{~mm}$ and 40 slices reconstructed at $1 \times 1 \times 2.5 \mathrm{~mm}$, FOV $230 \times 190 \times 100 \mathrm{~mm}$, acquisition time $7.5 \mathrm{~min})$ for MWF determination $(74,75)$.

\subsection{Neuropsychological testing}

The NIH Toolbox Cognition Battery (76) was used to evaluate cognition in both groups. The Test of Memory Malingering was used to exclude individuals showing suboptimal effort. Two primary composite cognitive measures were employed in this study (77). The Crystallized composite score (age adjusted) from the NIH Toolbox was used to assess crystallized cognitive ability, which refers to an accumulated storage of verbal knowledge and skills that are heavily influenced by educational and cultural experience. The crystallized score was based on the average performance on the Picture Vocabulary Test (language) and Oral Reading Recognition Test (language). The Fluid composite score was based on performance on the following NIH Toolbox measures: Dimensional Change Card Sort Test (attention, executive function), Flanker Inhibitory Control and Attention Test (attention, executive function), Picture Sequence Memory Test (episodic memory), Pattern Comparison Processing Speed Test (processing speed), List Sorting Working Memory Test (working memory). Fluid ability is defined as the capacity for new learning and information processing in novel situations, which is especially influenced by biological processes and is less dependent on past exposure. All scores were age-adjusted based on the NIH Toolbox nationally representative U.S. normative sample, and standard scores had a mean of 100 and standard deviation of 15 . Three healthy controls did not complete cognitive testing, one due to familiarity with the tests.

\subsection{Myelin water fraction (MWF) data analysis}

The signal decay curve obtained by the T2 relaxation sequence was modelled by multiple exponential components and the T2 distribution was estimated using in-house software in matlab (MathWorks, Massachusetts, U.S.A) which contained a regularized non-negative least squares algorithm using the extended phase graph and flip angle estimation to deal with stimulated echo artifacts $(29-31,78,79)$. MWF in each image voxel was computed as the ratio of the area under the T2 distribution with times of $10-40 \mathrm{~ms}$ to the total area under the distribution. MWF and 3DT1 images were registered to MNI space and brain extracted and segmented using tools from FSL (80-82). Slices below the inferior portion of the hypothalamus (mammillary body) were rejected in order to account for differing head sizes and make sure MWF was measured over the same brain coverage for all subjects. Global white matter (GWM) was segmented and pre-existing regions of interest from the JHU tract atlas in FSL (83-85) were used to segment specific tracts using FSL: splenium of corpus callosum (SCC), body of corpus callosum (BCC), genu of corpus callosum (GCC), minor forceps (MN), and right and left anterior 
183

184

185

186

187

188

189

190

191

192

193

194

195

196

197

198

199

200

201

202

203

204

205

206

207

208

209

210

211

212

213

214

215

216

217

218

219

220

221

222

223

224

225

226

227

228

thalamic radiation (ATR), inferior and superior longitudinal fasciculus (ILF and SLF respectively), and corticospinal tract (CST).

\subsection{Statistical analysis}

All statistical analysis was completed using IBM SPSS Statistics Version 25. Demographic differences were examined; age and education were compared between groups using an independent samples t-test and a chi-squared test was used to compare gender ratios between groups, where $p<0.05$ was considered significant. All group comparisons were done between controls and mild TBI, due to the low subject numbers in the severe TBI category. Mean MWF for ROIs were compared between participant groups using an independent samples t-test, where $\mathrm{p}<0.05$ was considered significant. Bonferroni-Holm correction for multiple comparisons was used to correct for multiple comparisons. The association between age and years of education and MWF in each ROI was examined using Pearson's correlations across controls and mTBI. A linear model comparing MWF (ANCOVA) across groups was used with age and education as covariates. Finally, cognitive scores were compared between groups using an independent sample's t-test and a Cohen's d to assess effect size. MWF was compared with cognitive scores in mTBI using Pearson's correlation.

\section{$3 \quad$ Results}

\subsection{Demographic characteristics between groups}

Table 1 shows the general group demographics and Supplementary Table S1 gives demographic and clinical features for all the participants in this study. Age, education and gender were not significantly different between controls and mild TBI.

\subsection{Myelin water fraction (MWF)}

Figure 1, meant for illustration purposes only, shows the MWF maps of six participants with TBI and six controls who were selected because they were closest in demographics. Note the overall qualitative reduction in MWF in each TBI participant. This was confirmed by a significant decrease in global white matter (GWM) MWF the mTBI group compared to controls (see first row of Table 2 and Figure 2). Analysis of the regions of interest (ROIs) for each group showed a significant decrease in MWF in the mTBI group compared with the controls in the majority of ROIs (see Figure 2 and Table 2), excluding the left anterior thalamic radiation (ATR) and right inferior longitudinal fasciculus (ILF) which were trending. After correcting for multiple comparisons using Bonferroni-Holm, only the splenium of corpus callosum (SCC) and left ILF were still significant. Due to the low subject numbers in the sTBI group it was not statistically compared.

The relationship between age and years of education and MWF across all controls and mTBI were evaluated using Pearson's correlations. Years of education was significantly positively correlated with MWF in all ROIs except in the SCC and left ILF and age was not significant in any ROI. Figure 3 shows MWF of global white matter in controls and mTBI plotted against years of education and age for each subject group. In the ANCOVA, group differences indicating lower GWM MWF in mTBI patients relative to controls were significant $\mathbf{p}=\mathbf{0 . 0 4}$ after removing variances accounted for by years of education, which had a significant effect on the model $(\mathbf{p}=\mathbf{0 . 0 1})$, and age, which did not $(\mathrm{p}=0.4)$. After controlling for education and age, the following ROIs still showed significant group differences: GCC $(\mathbf{p}=\mathbf{0 . 0 3}), \operatorname{SCC}(\mathbf{p}=\mathbf{0 . 0 1})$, SLFL $(\mathbf{p}=\mathbf{0 . 0 4})$, and SLFR $(\mathbf{p}=\mathbf{0 . 0 2})$, the other ROIs were not significant, $\mathrm{p}>0.05$. 


\subsection{Correlations between MWF and cognitive scores}

230 Average age adjusted cognitive composite scores for Crystallized and Fluid cognition are given in significantly higher in controls than mild TBI, and both means were above 100. Crystallized cognition was not significantly different between groups and both means were above 115 . The Cohen's $d$ suggested a moderate effect size for Crystallized and a large effect size for Fluid comparisons. Figure 5 shows the correlations between cognitive scores and GWM MWF for mTBI subjects. No significant correlations were found between mTBI MWF and cognitive scores in any ROI.

\section{Discussion}

In this study, we report several new findings in relation to MWF in chronic TBI. First, we found significantly lower MWF in chronic mild TBI as compared to matched age, gender and education healthy controls. Lower MWF was evident globally, and in most white matter regions examined, however only two ROIs survived analysis for multiple comparisons, SCC and left ILF. For global white matter the difference in MWF between mTBI and control was $12 \%$ - for the corpus callosum this decrease was $15-18 \%$. The consistent decrease in MWF in the participants with mTBI compared to controls in all areas examined is a strong result. The number of years of education had a significant effect on most ROI MWF measures but age did not. After accounting for the variance in MWF associated with differences in years of education, GCC, left ILF, SCC, SLF and global white matter MWF still exhibited significant group differences between controls and mTBI, indicating that the reduced MWF in patients was not solely due to education effects.

Severe TBI (sTBI) MWFs were generally lower than controls and mild TBI, however due to the low number of subjects in this category we did not able to statistically compare this group to the others. We would expect differences between controls and sTBI, as using a different technique to measure MWF, Choi et al.(70) also found significantly decreased MWF in a group with sTBI compared to healthy controls (these patients were only 2-months post-injury). However, the finding in mTBI is significant as most mTBI subjects are expected to return to pre-injury levels in terms of cognition and myelin in the chronic stage. For global white matter the difference in MWF between controls and mTBI was $12 \%$ - for the corpus callosum this decrease was 15.5-18\%. A previous MWF study(53) on athletes with concussion found a decrease in SCC MWF in the acute stage but return to baseline MWF at a month 2 follow up. We found the mTBI group was significantly lower than controls in global white matter, all three regions of the corpus callosum, MN, right ATR, left ILF, SLF and CST. There is previous evidence that a subset of people with mTBI report persistent symptoms and problems. It is likely that our group of participants highlights those with ongoing issues(64-69), as most participants in our study self-reported symptoms consistent with post-concussive syndrome. This is further supported by the fact that we found significant cognitive differences between controls and mTBI.

The corpus callosum connects the two hemispheres of the brain and thus is prone to the twisting and sheering motion during a TBI. The corpus callosum, and specifically the splenium of the corpus callosum is highlighted in TBI studies as a main area of change and injury (86). As well, the splenium is an area known to be rich in myelin and thus a high MWF (30). We found the CC, and specifically the SCC (significant after correction for multiple comparisons) sensitive to detecting differences between controls and mTBI. 
The exact pathway of myelin changes following TBI is not well characterized in humans, however, one post mortem human brain study found myelin loss (24). Many animal models have been developed to examine TBI (87-89). Disruptions of myelin sheaths have been found in stretching injury models of optic nerve in guinea pig $(90,91)$. Recent studies in rodent models have shown extensive demyelination $(22,23)$, remyelination but also, interestingly, extra myelin sheaths similar to redundant myelin which occurs during myelin development $(92,93)$. This pattern of demyelination, and excessive myelin may make it difficult to separate controls from TBI in early phases of TBI, as one would decrease and the other increase MWF. There is some evidence that clearance of myelin is slow and may further hinder axon/myelin repair $(94,95)$. However, there is also evidence of remyelination which would recover axonal signaling and functioning. Grin et al (25) reported that rats showed improved behavioral function following remyelination. Demyelinated axons are more vulnerable to damage, so ongoing loss of myelin can lead to further axonal damage, showing the importance of monitoring ongoing myelin changes post initial injury.

MWF has been found to change with age and years of education in earlier studies. Our study confirmed the relationship between MWF and years of education in healthy adults, however we also found this relationship in our TBI cohort. We did not find a significant correlation between age and MWF in either cohort or combined, which is different than what was found in the schizophrenia studies $(56,58)$. This is most likely due to the much lower mean age in the schizophrenia study. MWF changes are more dramatic at lower ages, while the increase of MWF with age is gradual in middle age (57), and thus the influence of age may not have been detectable in our cohort. These findings underline the importance of matching groups not only on age and gender but also years of education.

296

297

TBI subjects showed lower Crystallized and in particular Fluid cognitive scores than controls, suggesting decreased cognitive functioning in patients relative to controls. Despite this, it should be noted that the mean cognitive scores were higher than or equal to 100 (average range) for both groups, indicating that in this study we have a highly educated group of patients and controls. We did not find a significant correlation between measures of MWF and either crystallized or fluid cognition for mTBI. A recent study reported a significant association between global MWF and processing speed in a sample of patients with moderate to severe TBI at 3 months post-injury(70). We did not have a sufficient sample size in sTBI to evaluate relationships between cognitive scores and MWF. Persistent cognitive deficits are rarely found in mTBI; however, here we found lower cognitive scores in mTBI than controls, and this may be due to the select group of mTBI we studied who have persistent selfreported symptoms. Thus, the findings of this study may not be generalizable to patients with mild TBI who do not present with persisting symptoms.

Our TBI sample was highly educated and their mean CC score was above average which, may signal an elevated level of cognitive reserve. This high cognitive reserve may, limit the potential amount of cognitive morbidity, i.e. these patients may be able to tolerate more injury to the brain without it affecting their cognition. This reserve may prevent or restrict the observation of cognition-MWF associations(96). These associations would be predicted to be more evident in patients who were less educated or had less cognitive reserve.

Traumatic brain injury imparts a huge burden on society and even mild TBI can have long-term effects on patients. There is increasing evidence that TBI is not a 'single event' but rather an ongoing process which unfolds across months, years, and possibly over a lifetime; thus, improved monitoring of long- 
term effects of TBI is essential (97). White matter tracts are particularly vulnerable to a TBI and monitoring the myelination of these tracts may expand our knowledge and inform the development of diagnostic and prognostic biomarkers. Myelin water fraction is a powerful quantitative technique to examine myelin content and has been used to examine a variety of brain diseases and disorders (41). Here we show MWF differences in mTBI compared to healthy controls, months to decades post-injury, and show the utility of MWF to examine long-term effects of mild TBI.

\section{Acknowledgements}

This project is funded by MITACS in partnership with the Eaton-Arrowsmith group.

329

330

\section{$6 \quad$ Author Contributions}

I.T., W.P., A.L.M. and N.V.B. planned the study. B.R-S completed data analysis and prepared the manuscript. A.M.M. V.P. and L.B. managed the data collection. All authors contributed to the interpretation of the results and reviewed the manuscript.

\section{Conflict of Interest Statement}

Dr. Torres has served as consultant for Lundbeck Canada and Sumitomo Dainippon.

Dr. Panenka has a forensic psychiatry practice that focuses on head trauma.

Dr. Zhang is a GE clinical scientist but was not working with GE at the time she completed the research for this paper.

Dr. Virji-Babul: "No competing financial interests exist"

Dr. MacKay: "No competing financial interests exist"

Dr. Vavasour: "No competing financial interests exist"

Ms. Russell-Schulz: "No competing financial interests exist"

\section{References}

1. Sterr A, Herron KA, Hayward C, Montaldi D. Are mild head injuries as mild as we think ? Neurobehavioral concomitants of chronic post-concussion syndrome. 2006;10:1-10.

2. Stulemeijer M, Vos PE, Bleijenberg G, Werf SP Van Der. Cognitive complaints after mild traumatic brain injury: Things are not always what they seem. 2007;63:637-45.

3. Dikmen S, Machamer J, Fann JR, Temkin NR. Rates of symptom reporting following traumatic brain injury. 2017;(May):401-11.

4. Gupta R, Sen N. Traumatic brain injury: A risk factor for neurodegenerative diseases. Rev Neurosci. 2016;27(1):93-100.

5. Bigler ED, Green REA, Stern RA. Traumatic brain injury, neuroimaging, and neurodegeneration. 2013;

6. Faden AI, Loane DJ. Chronic Neurodegeneration After Traumatic Brain Injury: Alzheimer Disease, Chronic Traumatic Encephalopathy, or Persistent Neuroinflammation? Neurotherapeutics. 2015;12(1):143-50.

7. Koerte IK, Hufschmidt J, Muehlmann M, Lin AP, Shenton ME. Advanced Neuroimaging of Mild Traumatic Brain Injury. In: Laskowitz D GG, editor. Translational Research in Traumatic Brain Injury [Internet]. Boca Raton (FL): CRC Press/Taylor and Francis Group; 2016. Available from: https://www.ncbi.nlm.nih.gov/books/NBK326714

8. Yamamoto S, Levin HS, Prough DS. Mild, moderate and severe : terminology implications for clinical and experimental traumatic brain injury. 2018;31(6):672-80. 
9. Narayana PA, Yu X, Hasan KM, Wilde EA, Levin HS, Hunter J V., et al. Multi-modal MRI of mild traumatic brain injury. NeuroImage Clin [Internet]. 2015;7:87-97. Available from: http://dx.doi.org/10.1016/j.nicl.2014.07.010

10. Wintermark M, Sanelli PC, Anzai Y, Tsiouris AJ, Whitlow CT. Imaging Evidence and Recommendations for Traumatic Brain Injury: Advanced Neuro- and Neurovascular Imaging Techniques. 2015;1-11.

11. Jurick SM, Bangen KJ, Evangelista ND, Jak AJ, Bangen KJ, Evangelista ND. Advanced neuroimaging to quantify myelin in vivo : Application to mild TBI. Brain Inj [Internet]. 2017;30(12):1452-7. Available from: http://dx.doi.org/10.1080/02699052.2016.1219064

12. Lee B, Newberg A. Neuroimaging in traumatic brain imaging. NeuroRx. 2005;2(2):372-83.

13. Giza CC, Hovda DA. The Neurometabolic Cascade of Concussion. J Athl Train. 2001;36:22835.

14. Dixon KJ. Pathophysiology of Traumatic B rain Injury. Phys Med Rehabil Clin NA [Internet]. 2017;28(2):215-25. Available from: http://dx.doi.org/10.1016/j.pmr.2016.12.001

15. Prins M, Greco T, Alexander D, Giza CC. The pathophysiology of traumatic brain injury at a glance. Dis Model Mech [Internet]. 2013;6(September):1307-15. Available from: http://www.pubmedcentral.nih.gov/articlerender.fcgi?artid=3820255\&tool=pmcentrez\&renderty $\mathrm{pe}=$ abstract

16. Green RE a, Colella B, Maller JJ, Bayley M, Glazer J, Mikulis DJ. Scale and pattern of atrophy in the chronic stages of moderate-severe TBI. Front Hum Neurosci [Internet]. 2014;8(March):67. Available from: http://www.pubmedcentral.nih.gov/articlerender.fcgi?artid=3978360\&tool=pmcentrez\&renderty pe $=$ abstract

17. Adams JH, Doyle D, Fordt I, Gennarelli TA, Graham DI, Mclellan DR. Diffuse axonal injury in head injury : definition, diagnosis and grading. 1989;49-59.

18. Ng HK, Mahaliyana RD, Poon WS. The pathological spectrum of diffuse axonal injury in blunt head trauma: assessment with axon and myelin strains. Clin Neurol Neurosurg. 1994 Feb;96(1):24-31.

19. Tomaiuolo F, Bivona U, Lerch JP, Di M, Carlesimo GA, Ciurli P, et al. Memory and anatomical change in severe non missile traumatic brain injury : 1 vs . $\sim 8$ years follow-up. Brain Res Bull [Internet]. 2012;87(4-5):373-82. Available from: http://dx.doi.org/10.1016/j.brainresbull.2012.01.008

20. Shi H, Hu X, Leak RK, Shi Y, An C, Suenaga J, et al. Demyelination as a rational therapeutic target for ischemic or traumatic brain injury. Exp Neurol [Internet]. 2015 Oct;272:17-25. Available from: http://www.sciencedirect.com/science/article/pii/S0014488615000850

21. Tsunoda I, Fujinami SR. Inside-Out versus Outside-In models for virus induced demyelination: axonal damage triggering demyelination. Springer Semin Immunopathol [Internet]. 2002;24(2):105-25. Available from: http://dx.doi.org/10.1007/s00281-002-0105-z

22. Flygt J, Djupsjö a, Lenne F, Marklund N. Myelin loss and oligodendrocyte pathology in white matter tracts following traumatic brain injury in the rat. Eur J Neurosci [Internet]. 2013;38(1):2153-65. Available from: http://www.ncbi.nlm.nih.gov/pubmed/23458840

23. Mierzwa AJ, Marion CM, Sullivan GM, McDaniel DP, Armstrong RC. Components of myelin damage and repair in the progression of white matter pathology after mild traumatic brain injury. J Neuropathol Exp Neurol [Internet]. 2015;74(3):218-32. Available from: http://www.ncbi.nlm.nih.gov/pubmed/25668562

24. Johnson VE, Stewart JE, Begbie FD, Trojanowski JQ, Smith DH, Stewart W. Inflammation and 
white matter degeneration persist for years after a single traumatic brain injury. Brain.

413

414

415

416

417

418

419

420

421

422

423

424

425

426

427

428

429

430

431

432

433

434

435

436

437

438

439

440

441

442

443

444

445

446

447

448

449

450

451

452

453

454

455

456

457

2013;136(1):28-42.

25. Grin NM, Abdel-baki SG, Bergold PJ. Reversible Behavioral Deficits in Rats during a Cycle of Demyelination-Remyelination of the Fimbria. 2013;8(1).

26. Laule C, Vavasour IM, Kolind SH, Li DKB, Traboulsee TL, Moore GRW, et al. Magnetic Resonance Imaging of Myelin. Neurotherapeutics. 2007;4(3):460-84.

27. Weber AM, Torres C, Rauscher A. Imaging the Role of Myelin in Concussion. Neuroimaging Clin N Am [Internet]. 2018;28(1):83-90. Available from:

https://doi.org/10.1016/j.nic.2017.09.005

28. Alonso-Ortiz E, Levesque IR, Pike GB. MRI-Based Myelin Water Imaging : A Technical Review. 2015;81:70-81.

29. MacKay A, Whittall K, Adler J, Li D, Paty D, Graeb D. In vivo visualization of myelin water in brain by magnetic resonance. Magn Reson Med. 1994 Jun;31(6):673-7.

30. Whittall KP, MacKay AL, Graeb DA, Nugent RA, Li DK, Paty DW. In vivo measurement of T2 distributions and water contents in normal human brain. Magn Reson Med. 1997 Jan;37(1):3443.

31. MacKay A, Laule C, Vavasour I, Bjarnason T, Kolind S, Mädler B. Insights into brain microstructure from the T2 distribution. Magn Reson Imaging. 2006;24(4):515-25.

32. Swift T, Fritz, OG J. A proton spin-echo study of the state of water in frog nerves. Biophys J. 1969;9(1):54-9.

33. Vasilescu V, Katona E, Simplaceanu V DD. Water compartments in the myelinated nerve. III. Pulsed NMR results. Experientia. 1978;34(11):1443-4.

34. Webb S, Munro CA, Midha R, Stanisz GJ. Is Multicomponent T 2 a Good Measure of Myelin Content in Peripheral Nerve ? 2003;645(February 2002):638-45.

35. Stanisz GJ, Webb S, Munro CA, Pun T, Midha R. MR Properties of Excised Neural Tissue Following Experimentally Induced Inflammation. 2004;479:473-9.

36. Pun TWC, Odrobina E, Xu Q, Lam TJ, Munro CA, Midha R, et al. Histological and magnetic resonance analysis of sciatic nerves in the tellurium model of neuropathy. 2005;46:38-46.

37. Odrobina EE, Lam TYJ, Pun T, Midha R, Stanisz GJ. MR properties of excised neural tissue following experimentally induced demyelination. 2005;(June):277-84.

38. Valentine HL, Does MD, Marshall V, Tonkin EG, Valentine WM. Multicomponent T 2 analysis of dithiocarbamate-mediated peripheral nerve demyelination. 2007;28:645-54.

39. Menon RS, Allen PS. Application of continuous relaxation time distributions to the fitting of data from model systems and excised tissue. Magn Reson Med. 1991 Aug;20(2):214-27.

40. Gareau PP, Rutt BK, Bowen C V., Karlik SJ, Mitchell JR. In vivo Measurements of MultiComponent T2 Relaxation Behaviour in Guinea Pig Brain. 1999;17(9):1319-25.

41. Mackay AL, Laule C. Magnetic Resonance of Myelin Water : An in vivo Marker for Myelin. 2016;2:71-91.

42. Kozlowski P, Rosicka P, Liu J, Yung AC, Tetzlaff W. In vivo longitudinal Myelin Water Imaging in rat spinal cord following dorsal column transection injury is. Magn Reson Imaging [Internet]. 2014;32(3):250-8. Available from: http://dx.doi.org/10.1016/j.mri.2013.12.006

43. Kozlowski P, Liu J, Yung AC, Tetzlaff W. High-Resolution Myelin Water Measurements in Rat Spinal Cord. 2008;802:796-802.

44. Mccreary CR, Bjarnason TA, Skihar V, Mitchell JR, Yong VW, Dunn JF. NeuroImage Multiexponential T 2 and magnetization transfer MRI of demyelination and remyelination in murine spinal cord. Neuroimage [Internet]. 2009;45(4):1173-82. Available from: 
http://dx.doi.org/10.1016/j.neuroimage.2008.12.071

45. Laule C, Kozlowski P, Leung E, Li DKB, Mackay AL, Moore GRW. Myelin water imaging of multiple sclerosis at $7 \mathrm{~T}$ : Correlations with histopathology. 2008;40:1575-80.

46. Laule C, Leung E, Li DKB, Traboulsee AL, Paty DW, Mackay AL, et al. Myelin water imaging in multiple sclerosis : quantitative correlations with histopathology. 2006;(February).

47. Moore GR, Leung E, MacKay AL, Vavasour IM, Whittall KP, Cover KS, et al. A pathologyMRI study of the short-T2 component in formalin-fixed multiple sclerosis brain. Neurology. 2000 Nov;55(10):1506-10.

48. Laule C, Yung A, Pavolva V, Bohnet B, Kozlowski P, Hashimoto SA, et al. High-resolution myelin water imaging in post-mortem multiple sclerosis spinal cord : A case report. 2016;14859.

49. Laule C, Vavasour IM, Moore GRW, Oger J, Li DKB, Paty DW, et al. Water content and myelin water fraction in multiple sclerosis: A T 2 relaxation study. J Neurol. 2004;251(3):28493.

50. Levesque IR, Giacomini PS, Narayanan S, Ribeiro LT, Sled JG, Arnold DL, et al. Quantitative Magnetization Transfer and Myelin Water Imaging of the Evolution of Acute Multiple Sclerosis Lesions. 2010;640:633-40.

51. Faizy TD, Thaler C, Kumar D, Sedlacik J. Heterogeneity of Multiple Sclerosis Lesions in Multislice Myelin Water Imaging. 2016;1-13.

52. Oh J, Han ET, Lee MC, Nelson SJ, Pelletier D. Multislice Brain Myelin Water Fractions at $3 \mathrm{~T}$ in Multiple Sclerosis. 2007;156-63.

53. Wright AD, Jarrett M, Vavasour I, Shahinfard E, Kolind S, Van Donkelaar P, et al. Myelin water fraction is transiently reduced after a single mild traumatic brain injury - A prospective cohort study in collegiate hockey players. PLoS One. 2016;11(2):1-16.

54. Lebel C, Beaulieu C. Longitudinal development of human brain wiring continues from childhood into adulthood. J Neurosci. 2011 Jul;31(30):10937-47.

55. Yakovlev P, Lecours A. The myelogenetic cycles of regional maturation of the brain. In: Minkowski A, editor. Regional development of the brain in early life. Blackwell, Oxford; 1967. p. $3-70$.

56. Flynn SW, Lang DJ, MacKay AL, Goghari V, Vavasour IM, Whittall KP, et al. Abnormalities of myelination in schizophrenia detected in vivo with MRI, and post-mortem with analysis of oligodendrocyte proteins. Mol Psychiatry [Internet]. 2003;8(9):811-20. Available from: http://eutils.ncbi.nlm.nih.gov/entrez/eutils/elink.fcgi?dbfrom=pubmed\&id=12931208\&retmode= ref\&cmd=prlinks\%5Cnpapers2://publication/doi/10.1038/sj.mp.4001337

57. Arshad M, Stanley JA, Raz N. NeuroImage Adult age differences in subcortical myelin content are consistent with protracted myelination and unrelated to diffusion tensor imaging indices. Neuroimage [Internet]. 2016;143:26-39. Available from: http://dx.doi.org/10.1016/j.neuroimage.2016.08.047

58. Lang DJM, Yip E, Mackay AL, Thornton AE, Vila-Rodriguez F, Macewan GW, et al. 48 echo T2 myelin imaging of white matter in first-episode schizophrenia: Evidence for aberrant myelination. NeuroImage Clin [Internet]. 2014;6:408-14. Available from: http://dx.doi.org/10.1016/j.nicl.2014.10.006

59. Rabinowitz AR, Levin HS. Cognitive Sequelae of Traumatic Brain Injury. Psychiatr Clin NA [Internet]. 2014;37(1):1-11. Available from: http://dx.doi.org/10.1016/j.psc.2013.11.004

60. Dikmen S, Machamer J, Richard Winn H, R. Temkin N. Neuropsychological Outcome at 1-Year Post Head Injury. Vol. 9, Neuropsychology. 1995. 80-90 p. 
504

505

506

507

508

509

510

511

512

513

514

515

516

517

518

519

520

521

522

523

524

525

526

527

528

529

530

531

532

533

534

535

536

537

538

539

540

541

542

543

544

545

546

547

548

549

61. Schretlen DJ, Shapiro AM. International Review of Psychiatry A quantitative review of the effects of traumatic brain injury on cognitive functioning on cognitive functioning. 2009;0261.

62. Belanger HG, Curtiss G, Demery JA, Lebowitz BK, Vanderploeg RD. Factors moderating neuropsychological outcomes following mild traumatic brain injury : A meta-analysis. 2018;(2005):215-27.

63. Belanger HG, Vanderploeg RD. The neuropsychological impact of sports-related concussion: a meta-analysis. J Int Neuropsychol Soc. 2005 Jul;11(4):345-57.

64. Ghajar J, Johnson C, Kolster RA, Lee H, Meeker M, Zimmerman RD, et al. Extent of Microstructural White Matter Injury in Postconcussive Syndrome Correlates with Impaired Cognitive Reaction Time : A 3T Diffusion Tensor Imaging Study of Mild Traumatic Brain. 2008;(May):967-73.

65. Bazarian JJ, Wong T, Harris M, Leahey N, Mookerjee S, Dombovy M. Epidemiology and predictors of post-concussive syndrome after minor head injury in an emergency population. Brain Inj. 1999 Mar;13(3):173-89.

66. Deb S, Lyons I, Koutzoukis C. Neuropsychiatric sequelae one year after a minor head injury. 1998;899-902.

67. Masson F, Maurette P, Salmi LR, Dartigues J, Vecsey J, Erny P, et al. Prevalence of impairments 5 years after a head injury, and their relationship with disabilities and outcome. 2009;9052.

68. Bernstein DM. Information processing difficulty long after self-reported concussion. J Int Neuropsychol Soc. 2002 Jul;8(5):673-82.

69. Kraus MF, Susmaras T, Caughlin BP, Walker CJ, Sweeney JA, Little DM. White matter integrity and cognition in chronic traumatic brain injury: A diffusion tensor imaging study. Brain. 2007;130(10):2508-19.

70. Choi JY, Baek J-W, Lee J, Kim J. Evaluation of Myelin Damage in Diffuse Traumatic Brain Injury using ViSTa-MWI. In: ISMRM 25th Annual Meeting \& Exhibition, 22-27 April. Honolulu, HI, USA; 2017.

71. Porter S, Torres IJ, Panenka W, Rajwani Z, Fawcett D, Hyder A. Changes in brain-behavior relationships following a 3-month pilot cognitive intervention program for adults with traumatic brain injury. Heliyon [Internet]. 2017;(June):e00373. Available from: http://dx.doi.org/10.1016/j.heliyon.2017.e00373

72. Sheehan D V, Lecrubier Y, Sheehan KH, Ph D, D PAM, Ph D, et al. The Mini-International Neuropsychiatric Interview (M.I.N.I.): the development and validation of a structured diagnostic psychiatric interview for DSM-IV and ICD-10. 1998;59(suppl 20).

73. Workgroup NP, Diaz-arrastia R, Kochanek PM, Bergold P, Kenney K, Marx CE, et al. Pharmacotherapy of Traumatic Brain Injury : State of the Science and the Road Forward : Report of the Department of Defense. 2014;158:135-58.

74. Zhang J, Vavasour I, Kolind S, Baumeister R, Rauscher A, Mackay AL. Advanced Myelin Water Imaging Techniques for Rapid Data Acquisition and Long T2 Component Measurements. In: ISMRM 23rd Annual Meeting \& Exhibition,30 May-05 June. Toronto, Ontario, Canada; 2015.

75. Prasloski T, Rauscher A, MacKay AL, Hodgson M, Vavasour IM, Laule C, et al. Rapid whole cerebrum myelin water imaging using a 3D GRASE sequence. Neuroimage [Internet]. 2012;63(1):533-9. Available from: http://dx.doi.org/10.1016/j.neuroimage.2012.06.064

76. Weintraub S, Dikmen SS, Heaton RK, Tulsky DS, Zelazo PD, Bauer PJ, et al. Cognition assessment using the NIH Toolbox. Neurology [Internet]. 2013 Mar 12;80(11 Supplement 
3):S54-64. Available from:

http://www.neurology.org/content/80/11_Supplement_3/S54.abstract

77. Heaton RK, Akshoomoff N, Tulsky D, Mungas D, Dikmen S, Beaumont J, et al. Toolbox Cognition Battery in Adults. 2015;20(6):588-98.

78. Prasloski T, Ma B, Xiang Q, Mackay A, Jones C. Applications of Stimulated Echo Correction to Multicomponent T 2 Analysis. 2012;1814:1803-14.

79. Hennig J. Echoes - how to generate, recognize, use or avoid them in MR-imaging sequences.

Part II: Echoes in imaging sequences. Concepts Magn Reson [Internet]. 1991 Oct 1;3(4):179-92. Available from: http://dx.doi.org/10.1002/cmr.1820030402

80. Woolrich MW, Jbabdi S, Patenaude B, Chappell M, Makni S, Behrens T, et al. Bayesian analysis of neuroimaging data in FSL. Neuroimage [Internet]. 2009 Mar;45(1, Supplement 1):S173-86. Available from: http://www.sciencedirect.com/science/article/pii/S1053811908012044

81. Jenkinson M, Beckmann CF, Behrens TEJ, Woolrich MW, Smith SM. FSL. Neuroimage [Internet]. 2012 Aug 15;62(2):782-90. Available from: http://www.sciencedirect.com/science/article/pii/S1053811911010603

82. Smith SM, Jenkinson M, Woolrich MW, Beckmann CF, Behrens TEJ, Johansen-Berg H, et al. Advances in functional and structural MR image analysis and implementation as FSL. Neuroimage [Internet]. 2004;23, Supple:S208-19. Available from: http://www.sciencedirect.com/science/article/pii/S1053811904003933

83. Wakana S, Caprihan A, Panzenboeck MM, Fallon JH, Perry M, Gollub RL, et al. Reproducibility of Quantitative Tractography Methods Applied to Cerebral White Matter. Neuroimage [Internet]. 2007 Jul 1;36(3):630-44. Available from: http:/www.ncbi.nlm.nih.gov/pmc/articles/PMC2350213/

84. Hua K, Zhang J, Wakana S, Jiang H, Li X, Reich DS, et al. Tract Probability Maps in Stereotaxic Spaces: Analyses of White Matter Anatomy and Tract-Specific Quantification. Neuroimage [Internet]. 2008 Jan 1;39(1):336-47. Available from: http:/www.ncbi.nlm.nih.gov/pmc/articles/PMC2724595/

85. Susumu Mori, S. Wakana, Peter C M van Zijl LMN-P. MRI Atlas of Human White Matter. annotated. Elsevier; 2005. 276 p.

86. Narayana PA. White matter changes in patients with mild traumatic brain injury : MRI perspective. 2017;

87. Xiong Y, Mahmood A, Chopp M. Animal models of traumatic brain injury. Nat Rev Neurosci [Internet]. 2013;14(2):128-42. Available from: http://www.nature.com/doifinder/10.1038/nrn3407

88. Francisco E-R, Jesús M-TR, Francisco E-B, Marina M-V, Adán P-A, Luis F-A, et al. Models used in the study of traumatic brain injury [Internet]. Vol. 0, Reviews in the Neurosciences. 2017. Available from: https:/www.degruyter.com/view/j/revneuro.ahead-of-print/revneuro2017-0028/revneuro-2017-0028.xml

89. Johnson VE, Meaney DF, Cullen DK, Smith DH. Animal models of traumatic brain injury. 2016;127:115-28.

90. Maxwell WL, Domleo A, Mccoll G, Jafari SS, Graham DI, Al MET. Post-Acute Alterations in the Axonal Cytoskeleton after Traumatic Axonal Injury. 2003;20(2):151-68.

91. William L, Brian J, Graham DI, Al MET. Freeze-Fracture and Cytochemical Evidence for Structural and Functional Alteration in the Axolemma and Myelin Sheath of Adult Guinea Pig Optic Nerve Fibers After Stretch Injury. 1999;16(4):273-84. 
601

602

603

604

605

606

607

608

609

610

611

612

613

614

615

616

617

618

619

620

621

622

623

624

625

626

627

628

629

630

631

632

633

634

635

636

637

638

639

92. Armstrong RC, Mierzwa AJ, Sullivan GM, Sanchez MA. Myelin and oligodendrocyte lineage cells in white matter pathology and plasticity after traumatic brain injury. Neuropharmacology [Internet]. 2015; Available from: http://dx.doi.org/10.1016/j.neuropharm.2015.04.029

93. Armstrong RC, Mierzwa AJ, Marion CM, Sullivan GM. White matter involvement after TBI: Clues to axon and myelin repair capacity. Experimental Neurology. 2016.

94. Vargas ME, Barres BA. Why Is Wallerian Degeneration in the CNS So Slow ? 2007;

95. Wen L, Xu J, Zhan T, Wang H, Huang X, Liu W, et al. The occurrence of diffuse axonal injury in the brain: associated with the accumulation and clearance of myelin debris. Neural Regen Res [Internet]. 2014 Nov 1;9(21):1902-6. Available from: https://www.ncbi.nlm.nih.gov/pubmed/25558240

96. Steward KA, Kennedy R, Novack TA, Crowe M, Marson DC, Triebel KL. The Role of Cognitive Reserve in Recovery from Traumatic Brain Injury. J Head Trauma Rehabil. 2018;33(1):E18-27.

97. Masel BE, Dewitt DS. Traumatic Brain Injury : A Disease Process, Not an Event. 2010;1540(August):1529-40.

\section{$9 \quad$ Data availability statement}

The UBC Ethics Board will not allow us to store data outside of Canada, however if there are researchers interested in using the data we can apply for an amendment to share anonymized data with individual institutions.

Information on implementing the myelin water imaging technique and analysis code is available on github via a request form through the UBC MRI Research Centre website (https://mriresearch.med.ubc.ca/news-projects/myelin-water-fraction/).

\section{Figure Legends}

Figure 1: Sample myelin water fraction (MWF) maps for control and TBI participants.

Figure 2: Myelin water fraction (MWF) for all controls and TBI subjects; in all regions of interest; genu of corpus callosum (GCC), body of corpus callosum (BCC), splenium of corpus callosum (SCC), minor forceps (MN), global white matter (GWM), left and right anterior thalamic radiation (ATR), superior and inferior longitudinal fasciculus (SLF and ILF respectively) and corticospinal tract (CST). Independent t-test comparison between control and mTBI uncorrected p-values are represented as $*<0.05, * *<0.01$ and are taken from Table 2 .

Figure 3: Effect of years of education and age on myelin water fraction (MWF) of global white matter across controls and mild TBI. The linear fit over controls and mTBI is represented by the black dashed line and the Pearson's correlation coefficient $(r)$ and p-value between education/age and MWF are reported.

Figure 4: Age adjusted Fluid and Crystallized Composite scores for each subject group and subject, and independent t-test comparison between control and mTBI p-values are represented as $*<0.05$. Figure 5: Age adjusted Fluid and Crystallized Composite scores compared to global white matter myelin water fraction (MWF) for mild TBI participants. Pearson's correlation coefficient $(r)$ and pvalue between are reported.

\section{Tables}


640 Table 1: Subject demographics; where TBI = Traumatic Brain Injury and $\mathrm{m}=$ mild and $\mathrm{s}=$ severe. Age 641 642 and education compared between controls and mTBI using an independent samples t-test and a chisquared test to compare group gender ratios

\begin{tabular}{|c|c|c|c|c|c|c|c|}
\hline $\begin{array}{l}\text { Subject } \\
\text { Group }\end{array}$ & $\mathrm{N}$ & \multicolumn{2}{|c|}{$\begin{array}{l}\text { Gender Ratio } \\
(\mathrm{M}: \mathrm{F})\end{array}$} & \multicolumn{2}{|l|}{$\begin{array}{l}\text { Age (years) } \\
\text { Mean } \pm \text { sd (Range) }\end{array}$} & \multicolumn{2}{|c|}{$\begin{array}{l}\text { Education (years) } \\
\text { Mean } \pm \text { sd (Range) }\end{array}$} \\
\hline Control & 12 & $8: 4$ & \multirow[t]{2}{*}{$\mathrm{p}=0.8$} & $37.2 \pm 12(21-53)$ & \multirow[t]{2}{*}{$\mathrm{p}=0.8$} & $16.4 \pm 3(12-20)$ & \multirow[t]{2}{*}{$\mathrm{p}=0.2$} \\
\hline mTBI & 18 & $9: 9$ & & $38.5 \pm 12(18-57)$ & & $15.5 \pm 2(12-18)$ & \\
\hline sTBI & 4 & \multicolumn{2}{|l|}{$3: 1$} & \multicolumn{2}{|l|}{$47.5 \pm 6(40-54)$} & \multicolumn{2}{|l|}{$14.5 \pm 2(12-18)$} \\
\hline
\end{tabular}

643 Table 2: Mean and standard error of myelin water fraction (MWF) of all regions of interest; global 644 white matter (GWM), splenium of corpus callosum (SCC), body of corpus callosum (BCC), genu of 645 corpus callosum (GCC), anterior thalamic radiation (ATR), minor forceps (MN), inferior and superior 646 longitudinal fasciculus (ILF and SLF respectively), corticospinal tract (CST) for controls and mTBI 647 648 compared with an independent sample's t-test. P-values below 0.05 are bolded and those that survive Holm-Bonferroni analysis for multiple comparisons are starred.

\begin{tabular}{|c|c|c|c|c|}
\hline \multicolumn{2}{|c|}{ Region of Interest } & Control $(n=12)$ & Mild TBI $(n=18)$ & p-value \\
\hline \multicolumn{2}{|c|}{ GWM } & $0.114 \pm 0.005$ & $0.101 \pm 0.003$ & 0.02 \\
\hline \multicolumn{2}{|l|}{$\mathrm{SCC}$} & $0.147 \pm 0.006$ & $0.125 \pm 0.004$ & $0.004 *$ \\
\hline \multicolumn{2}{|l|}{$\mathrm{BCC}$} & $0.111 \pm 0.006$ & $0.095 \pm 0.004$ & 0.02 \\
\hline \multicolumn{2}{|l|}{$\mathrm{GCC}$} & $0.103 \pm 0.005$ & $0.086 \pm 0.004$ & 0.01 \\
\hline \multicolumn{2}{|l|}{$\mathrm{MN}$} & $0.087 \pm 0.006$ & $0.074 \pm 0.002$ & 0.02 \\
\hline \multirow[t]{2}{*}{ ATR } & Left & $0.122 \pm 0.006$ & $0.109 \pm 0.004$ & 0.05 \\
\hline & Right & $0.112 \pm 0.006$ & $0.097 \pm 0.003$ & 0.03 \\
\hline \multirow[t]{2}{*}{ ILF } & Left & $0.100 \pm 0.005$ & $0.079 \pm 0.004$ & $0.003 *$ \\
\hline & Right & $0.133 \pm 0.006$ & $0.120 \pm 0.004$ & 0.05 \\
\hline \multirow[t]{2}{*}{ SLF } & Left & $0.131 \pm 0.005$ & $0.115 \pm 0.004$ & 0.02 \\
\hline & Right & $0.144 \pm 0.006$ & $0.126 \pm 0.003$ & 0.01 \\
\hline \multirow[t]{2}{*}{ CST } & Left & $0.205 \pm 0.006$ & $0.189 \pm 0.004$ & 0.03 \\
\hline & Right & $0.194 \pm 0.006$ & $0.178 \pm 0.004$ & 0.03 \\
\hline
\end{tabular}

649 Table 3: Mean and sd of age-adjusted cognitive scores for controls and TBI with MRIs compared using 650 an independent t-test and a Cohen's d to measure effect size.

\begin{tabular}{|c|c|c|c|c|c|c|c|}
\hline \multirow[t]{2}{*}{$\begin{array}{l}\text { Subject } \\
\text { Group }\end{array}$} & \multirow[t]{2}{*}{$\mathrm{N}$} & \multicolumn{3}{|c|}{$\begin{array}{l}\text { Crystallized Cognition Composite } \\
\text { Score }\end{array}$} & \multicolumn{3}{|c|}{ Fluid Cognition Composite Score } \\
\hline & & Mean (sd) & p-value & Cohen's d & Mean (sd) & $\mathrm{p}$-value & Cohen's d \\
\hline Control & 9 & $123(5)$ & \multirow[t]{2}{*}{0.09} & \multirow[t]{2}{*}{0.52} & $117(12)$ & \multirow[t]{2}{*}{0.01} & \multirow[t]{2}{*}{1.1} \\
\hline $\mathrm{mTBI}$ & 18 & $116(11)$ & & & $\begin{array}{l}101(17) \\
n=17\end{array}$ & & \\
\hline sTBI & 4 & \multicolumn{3}{|l|}{$107(11)$} & \multicolumn{3}{|l|}{$85(6)$} \\
\hline
\end{tabular}




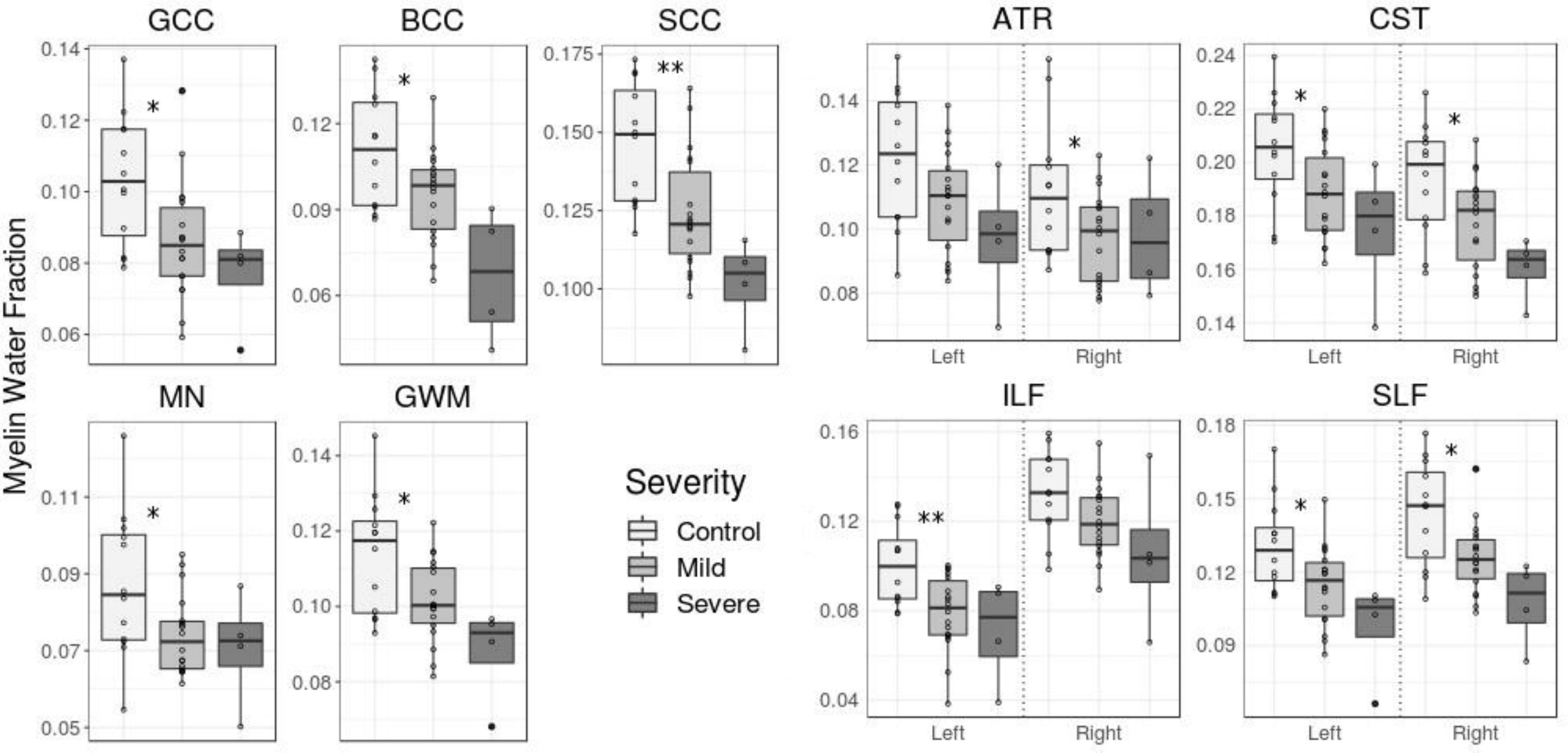




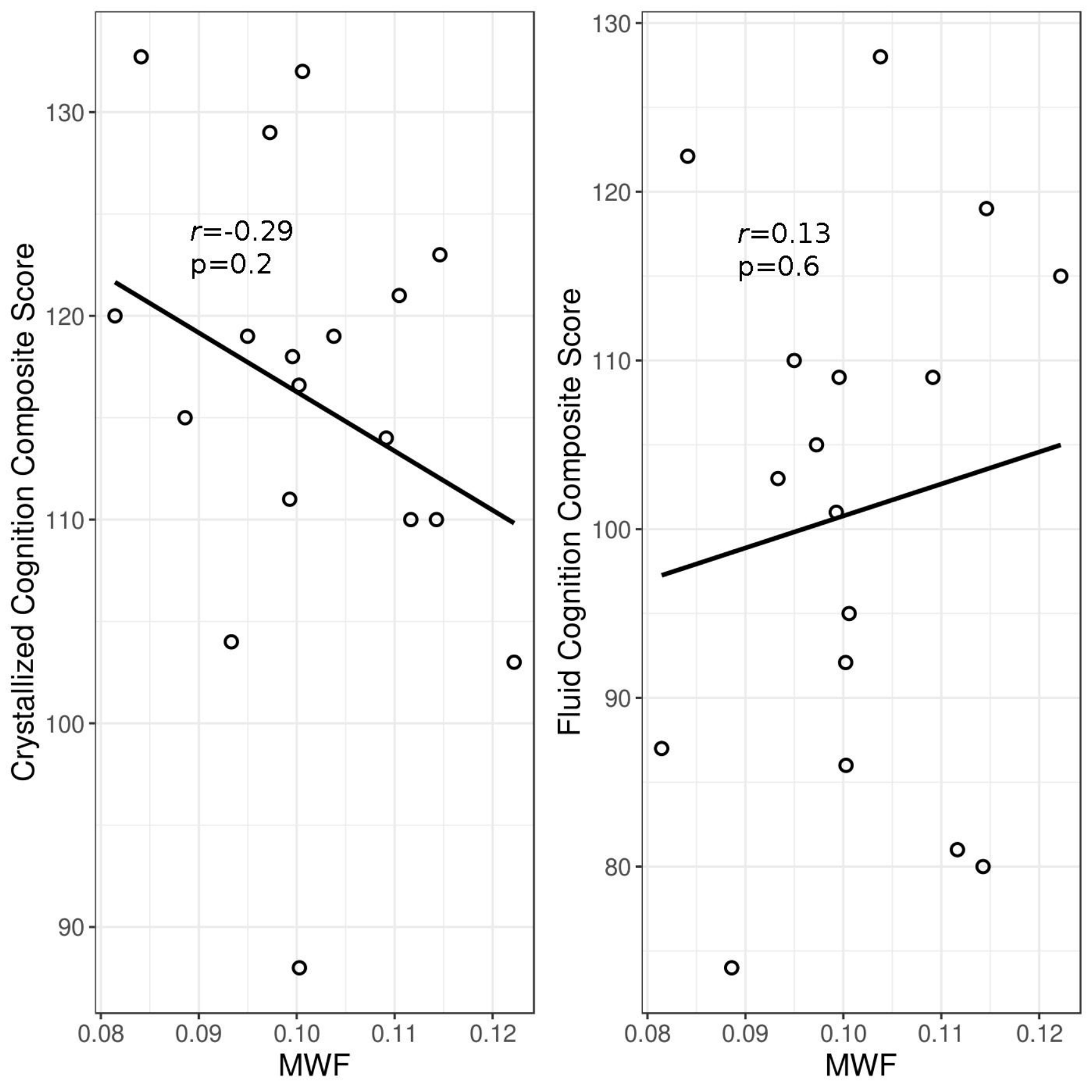

